\title{
Complex epsilon-near-zero metamaterials for broadband light harvesting systems
}

\section{Marcella Bonifazi, Yi Tian, Andrea Fratalocchi}

Marcella Bonifazi, Yi Tian, Andrea Fratalocchi, "Complex epsilon-near-zero metamaterials for broadband light harvesting systems," Proc. SPIE 10527, Physics, Simulation, and Photonic Engineering of Photovoltaic Devices VII, 1052710 (16 February 2018); doi: 10.1117/12.2289977

SPIE. Event: SPIE OPTO, 2018, San Francisco, California, United States 


\title{
Complex epsilon-near-zero metamaterials for broadband light harvesting systems
}

\author{
Marcella Bonifazi ${ }^{a}$, Yi $\operatorname{Tian}^{a}$ and Andrea Fratalocchi ${ }^{a}$ \\ ${ }^{a}$ PRIMALIGHT, King Abdullah University of Science and Technology (KAUST), Thuwal \\ 23955-6900, Saudi Arabia;
}

\begin{abstract}
We engineered an epsilon-near-zero (ENZ) material from suitably disordered metallic nanostructures. We create a new class of dispersionless composite materials that efficiently harnesses white light. By means of Atomic Force Microscopy (AFM) and Photoluminescence (PLE) measurements we experimentally demonstrate that this nanomaterial increases up to a record value the absorption of ultra-thin light harvesting films at visible and infrared wavelengths. Moreover, we obtained a $170 \%$ broadband increase of the external quantum efficiency (EQE) when these ENZ materials are inserted in an energy-harvesting module. We developed an inexpensive electrochemical deposition process that enables large-scale production of this material for energy-harvesting applications.
\end{abstract}

Keywords: Epsilon Near Zero, Metamaterials, energy harvesting, plasmonics, light localization, large-scale fabrication

\section{INTRODUCTION}

Recently, many efforts have been devoted to enhance the performances of light harvesting systems. In this context Colloidal quantum dot (CQD) films are often used in a wide array of energy harvesting devices, ranging from photodetectors ${ }^{1-4}$ to photovoltaic devices ${ }^{5-10}$ and photoJFET devices. ${ }^{11}$ These materials show very interesting characteristics such as from cost-effective fabrication and low material utilization, to size-tunable optical bandgap. Altogether, these features enable the chance to engineer the spectral response over a wide of range of wavelength within a low-cost platform. ${ }^{12}$

Unfortunately a strong restraint which is preventing this technology from improving is the compromise between charge carrier extraction and light absorption. Even though more than $1 \mu \mathrm{m}$ of planar material is required to absorb all photons with wavelengths greater than $800 \mathrm{~nm}$, transport lengths are limited to $\approx 400$ $\mathrm{nm} .{ }^{12}$ In the last few years numerous strategies have been developed to address this topic, from engineering bulk or depleted heterojunction in such a way to reduce the collection distance ${ }^{7,13-15}$ to micro and nano-structuring substrates in order to couple light directly in the active layer. ${ }^{16,17}$ One of the most promising approach is to use plasmonic nanostructures incorporated in the CQD film to obtain an enhancement of near field absorption but the main drawback is that most of the energy is usually localized inside the metallic material. ${ }^{18}$

The fundamental challenge is to develop a material that could capture incoming photons with energy above $1.1 \mathrm{eV}$ to achieve the thermodynamic limit of efficiency for a single junction solar cell. ${ }^{19}$

Here we present a new class of broadband epsilon-near zero (ENZ) materials based on disordered metallic plasmonic nanostructures, which localize broadband radiation within ultra-thin light harvesting films, greatly enhancing their absorption. ${ }^{20}$ This happens because, during propagation inside the material, the phase velocity of light diverges and the energy velocity tends to zero. As a consequence standing waves with infinite wavelength are formed. Thus, a broadband ENZ nanomaterial could slow down electromagnetic radiation on scales smaller than the charge diffusion length. This could pave the way to the design of a new class of ultra-thin energy harvesting system, reducing the thickness of the layer of the active material needed to build the device.

Exploiting complex metallic nanostructures we design an innovative light harvesting device based on ultrabroadband ENZ material. This nanoplasmonic material is able to form a complex network of all dielectric ENZ

Further author information: (Send correspondence to Andrea Fratalocchi)

Andrea Fratalocchi: E-mail: andrea.fratalocchi@kaust.edu.sa

Physics, Simulation, and Photonic Engineering of Photovoltaic Devices VII, edited by Alexandre Freundlich,

Laurent Lombez, Masakazu Sugiyama, Proc. of SPIE Vol. 10527, 1052710

(c) 2018 SPIE · CCC code: 0277-786X/18/\$18 · doi: 10.1117/12.2289977

Proc. of SPIE Vol. 10527 1052710-1 


\section{a)}

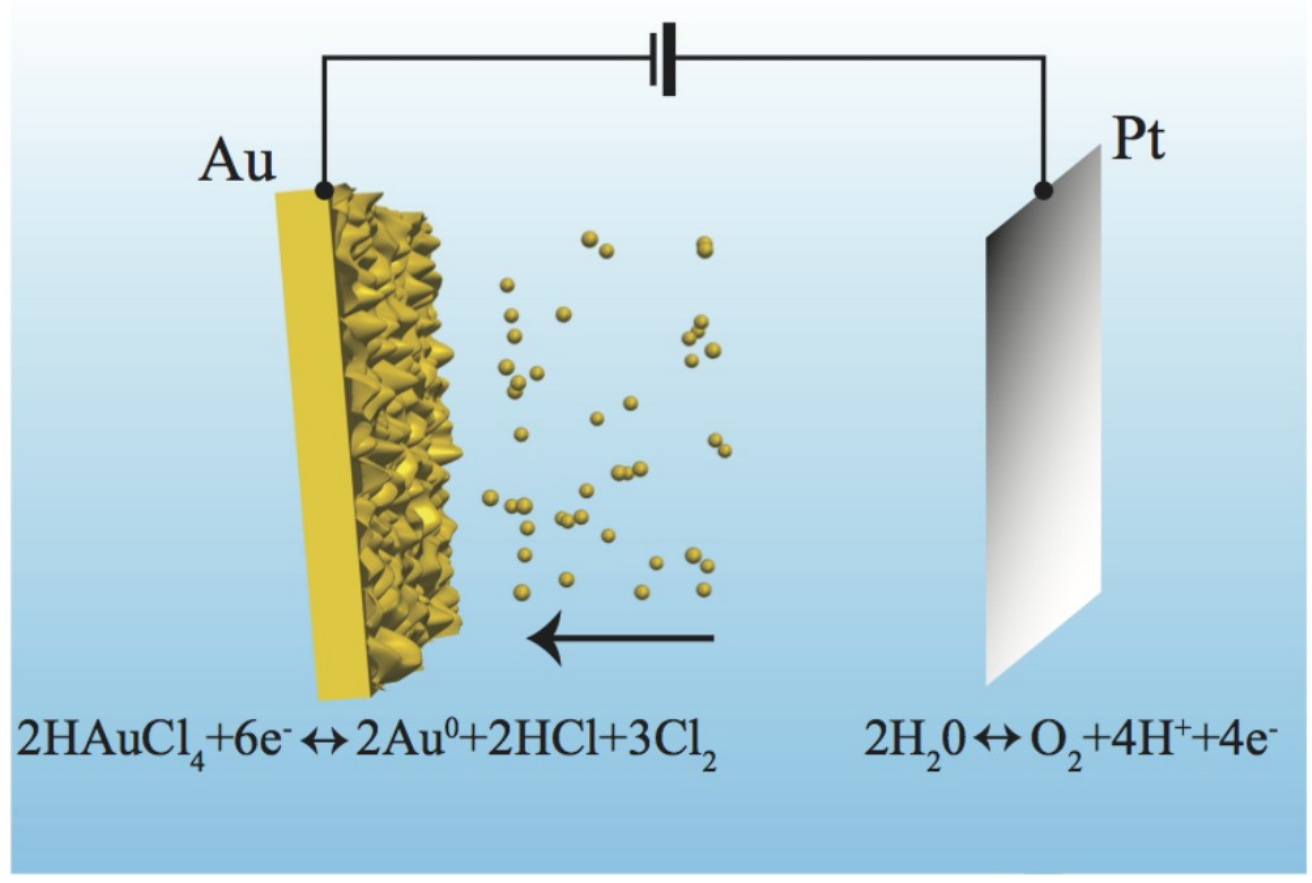

Figure 1. Fabrication process from Epsilon Near Zero Metamaterials

nanostructures combined with very high dielectric permittivities nanocavities. As a result, we efficiently localize white light within $10-100 \mathrm{~nm}$ outside the metal, developing a material which can be easily combined with ultra-thin absorptive film, achieving very high efficiency in light absorption.

\section{MATERIAL DESIGN AND ABSORPTION RESULTS}

A complex ENZ material can be engineered from a suitable assembly of random metallic structures. It is therefore important to develop a fabrication technique whereby disorder can be produced on large scales and with controllable features. In order to assemble a broadband ENZ nanomaterial, we used an electroplating process that grows a random networks of gold $(\mathrm{Au})$ nanowires from a flat metallic Au substrate ${ }^{32-35}$ (Figure 1). Figure 2a shows SEM cross sections of a fabricated sample. By using an exact analysis based on transformation optics, we demonstrate that the complex metallic network of Fig. 2a is exactly equivalent to a flat metallic panel with a series of ENZ nano-regions on top (Fig. 2c), connected by areas of high dielectric constant. This analysis is based on the introduction of a conformal grid $(u, v)$, illustrated in Fig. 2b, which describes the propagation of surface plasmons polariton (SPP) waves excited at the air/metal interface of the nanowires. The ENZ regions are created in proximity of the metallic peaks of the nanostructures and are completely dispersionless, existing at every wavelength.

When a thin layer of absorptive material is deposited on top of the ENZ nanostructures, we show that incoming radiation gets trapped inside the ENZ material, originating a quasi-static field localized in areas of $10-100$ $\mathrm{nm}$ in proximity of the metal. In a series of AFM, Photoconductive AFM (PC-AFM) and Photoluminescence excitation (PLE) measurements, which are illustrated in Fig. 2, we investigate the impact of ENZ nanostructures on the broadband absorption of an ultra-thin CQD film of 50-300 nm thickness. Figure 2a reports the PC-AFM map of one region of a sample. The spatial current distribution is illustrated as a pseudocolor plot in Fig. 2a, and is superimposed on the surface topography of the sample, which is obtained by AFM. From the combined AFM and PC-AFM results, we observe a great current enhancement on the peak of the structures, where the ENZ regions are formed. Figures 3a-b report the time dynamics of the photocurrent measured by the PC-AFM 
a)

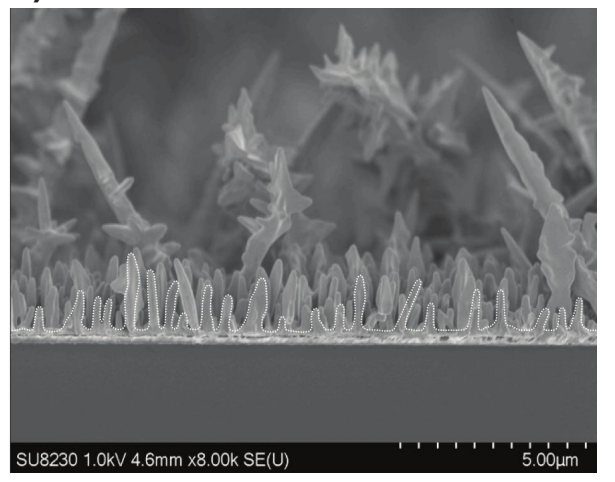

b)

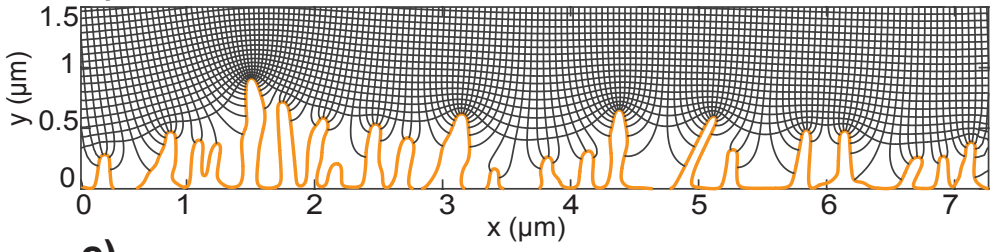

c)

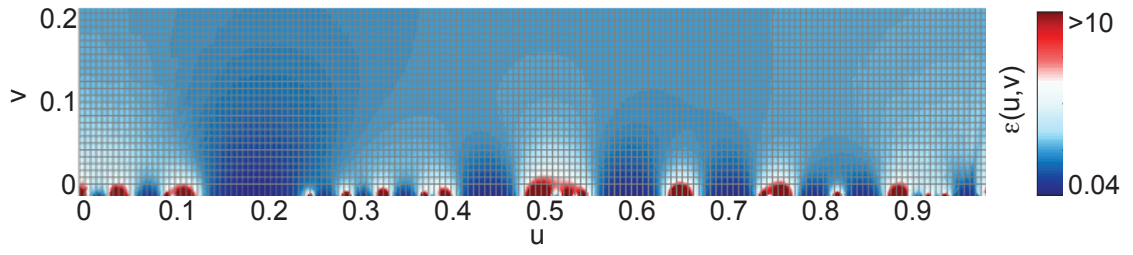

Figure 2. ENZ materials design: a) SEM cross section of a nanostructured sample, b) 2D reconstructed cross section of the profile, c) equivalent ENZ representation of the random nanowires illustrated in panel b.

on peaks (Fig. 3a) and valleys (Fig. 2b) of the sample, when the input laser is periodically switched on and off. These measurements demonstrate that the ENZ regions are responsible for a significant absorption increase in the structure. Figure 2d, finally, shows the results of PLE experiments, which measure the absorption increase in the CQD region.

For CQD thicknesses between $50-300 \mathrm{~nm}$, we experimentally observed a dramatic absorption enhancement $(\approx 400 \%)$ when compared to the PLE signal from an equivalent planar configuration (Fig. 2d, dashed line), which becomes completely flat in the visible and infrared regions.

\section{CONCLUSIONS}

Herein we introduced a new class of ENZ nanomaterials that allow broadband harvesting of light in ultra-thin CQD films. This is made possible by the use of a suitable optical transformation from complex disordered metallic materials. We verified our theoretical model trough different experimental measurements, such as AFM and spatial photoluminescence efficiency measurements. We demonstrated a dramatic increase in the absorption capability inside the active film. Furthermore, experiments on an ENZ based solar harvesting device performed in a range between $\mathrm{UV}(\lambda=400) \mathrm{nm}$ and infrared $(\lambda=1200 \mathrm{~nm})$ radiation reported a broadband, $170 \%$ quantum efficiency increase if compared to an identical planar structure without ENZ . In addition, the lightmatter interaction described in this work is a general effect that does not rely on the type of absorptive medium employed. This constitutes a great advantage since it means that it could be generalized also for other common and largely used semiconductors films, such as Si. The versatility of this platform and the easy fabrication process result in a completely new nanostructured material that represents a significant progress in the enhancement of the efficiency of CQD optoelectronics and could serve as a fundamental point for the development process of a new generation of photovoltaic devices. 
a)

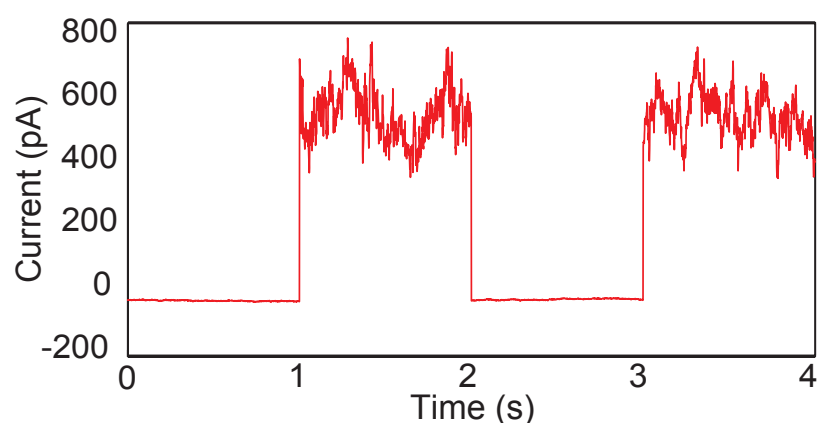

b)

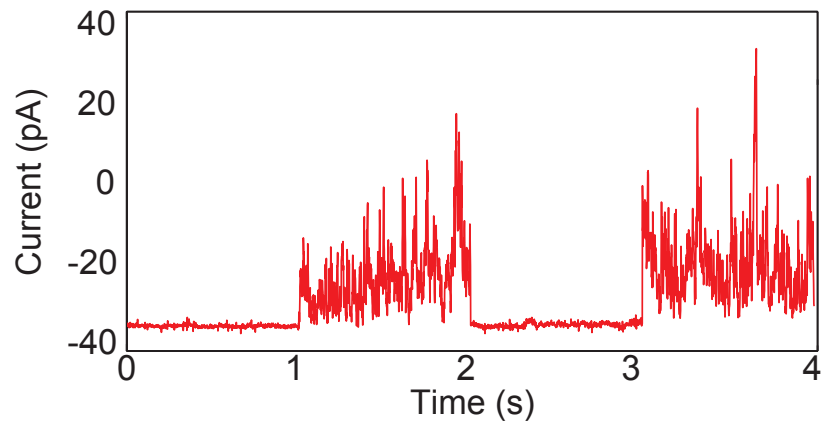

c)
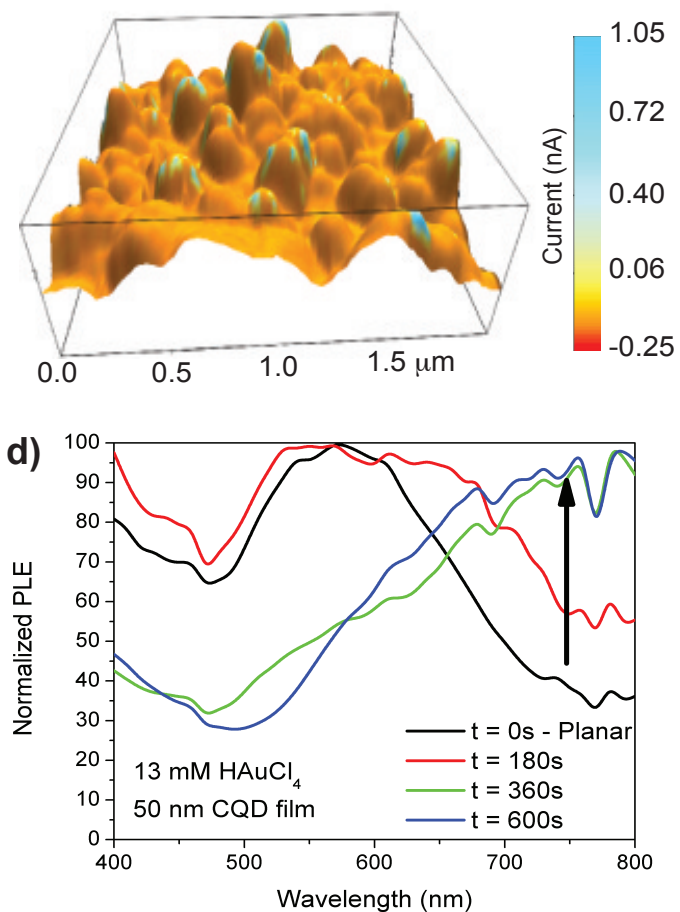

Figure 3. Light trapping in ENZ nanostructures: photoconductive AFM and PLE measurements. a)-b) Photocurrent obtained at $\mathrm{V}=0$ by periodically switching on and off light from a laser source, peak and valley position respectively. c) Photocurrent distribution plotted over surface profile. d) PLE signals of 50-300 nm CQD film deposited on ENZ nanostructures.

\section{REFERENCES}

1. Oertel, D. C., Bawendi, M. G., Arango, A. C. \& Bulović, V. Photodetectors based on treated cdse quantumdot films. App. Phys. Lett 87, 213505-3 (2005).

2. Clifford, J. P. et al. Fast, sensitive and spectrally tuneable colloidal-quantum-dot photodetectors. Nature Nanotechnology 4, 40-44 (2009).

3. Konstantatos, G. et al. Ultrasensitive solution-cast quantum dot photodetectors. Nature 442, 180-183 (2006).

4. Konstantatos, G. et al. Hybrid graphenequantum dot phototransistors with ultrahigh gain. Nature Nanotechnology 7, 363-368 (2012).

5. Chuang, C.-H. M., Brown, P. R., Bulović, V. \& Bawendi, M. G. Improved performance and stability in quantum dot solar cells through band alignment engineering. Nature Materials 13, 796-801 (2014).

6. Ip, A. H. et al. Hybrid passivated colloidal quantum dot solids. Nature Nanotechnology 7, 577-582 (2012).

7. Rath, A. K. et al. Solution-processed inorganic bulk nano-heterojunctions and their application to solar cells. Nature Photonics 6, 529-534 (2012).

8. Choi, J. J. et al. Solution-processed nanocrystal quantum dot tandem solar cells. Advanced Nanomaterials 23, 3144-3148 (2011).

9. Pattantyus-Abraham, A. G. et al. Depleted-heterojunction colloidal quantum dot solar cells. ACS Nano 4, 3374-3380 (2010).

10. Jean, J. et al. Zno nanowire arrays for enhanced photocurrent in pbs quantum dot solar cells. Advanced Materials 25, 2790-2796 (2013).

11. Adinolfi, V. et al. Photojunction field-effect transistor based on a colloidal quantum dot absorber channel layer. ACS Nano 9, 356-362 (2015). 
12. Labelle, A. J. et al. Conformal fabrication of colloidal quantum dot solids for optically enhanced photovoltaics. ACS Nano 9, 5447-5453 (2015).

13. Barkhouse, D. A. R. et al. Depleted bulk heterojunction colloidal quantum dot photovoltaics. Advanced Materials 23, 3134-3138 (2011).

14. Lan, X. et al. Self-assembled, nanowire network electrodes for depleted bulk heterojunction solar cells. Advanced Materials 25, 1769-1773 (2013).

15. Kramer, I. J. et al. Ordered nanopillar structured electrodes for depleted bulk heterojunction colloidal quantum dot solar cells. Advanced Materials 24, 2315-2319 (2012).

16. Adachi, M. M. et al. Broadband solar absorption enhancement via periodic nanostructuring of electrodes. Scientific Report 3 (2013).

17. Labelle, A. J. et al. Colloidal quantum dot solar cells exploiting hierarchical structuring. Nano Letters 15, 1101-1108 (2015).

18. Paz-Soldan, D. et al. Jointly tuned plasmonicexcitonic photovoltaics using nanoshells. Nano Letters 13, 1502-1508 (2013).

19. Shockley, W. \& Queisser, H. J. Detailed balance limit of efficiency of p-n junction solar cells. Journal of Applied Physics 32, 510-519 (1961).

20. Labelle, A. et al. Broadband epsilon-near-zero reflectors enhance the quantum efficiency of thin solar cells at visible and infrared wavelengths. ACS Applied Materials and Interfaces 9, 5556-5565 (2017).

21. Silveirinha, M. \& Engheta, N. Tunneling of electromagnetic energy through subwavelength channels and bends using epsilon-near-zero materials. Phys. Rev. Lett. 97, 157403 (2006).

22. Maas, R., Parsons, J., Engheta, N. \& Polman, A. Experimental realization of an epsilon-near-zero metamaterial at visible wavelengths. Nat Photonics 7, 907-912 (2013).

23. Vesseur, E. J. R., Coenen, T., Caglayan, H., Engheta, N. \& Polman, A. Experimental verification of $n=0$ structures for visible light. Phys. Rev. Lett. 110, 013902 (2013).

24. Liu, C. et al. Enhanced energy storage in chaotic optical resonators. Nature Photon. 7, 473-478 (2013).

25. Conti, C., Leonetti, M., Fratalocchi, A., Angelani, L. \& Ruocco, G. Condensation in disordered lasers: Theory, simulations, and experiments. Phys. Rev. Lett. 101, 143901-(4) (2008).

26. Liu, C. et al. Triggering extreme events at the nanoscale in photonic seas. Nature Phys. 11, 358-363 (2015).

27. Conti, C. \& Fratalocchi, A. Dynamic light diffusion, three-dimensional anderson localization and lasing in inverted opals. Nature Phys. 4, 794-798 (2008).

28. Stockman, M. I. Chaos and spatial correlations for dipolar eigenproblems. Phys. Rev. Lett. 79, 4562-4565 (1997).

29. Shalaev, V. M. Nonlinear Optics of Random Media: Fractal Composites and Metal-Dielectric Films (Springer, Berlin, 2013).

30. Huang, J. et al. Harnessing structural darkness in the visible and infrared wavelengths for a new source of light. Nature Nanotechnology 11, 60-66 (2016).

31. Where now for plasmonics? Nat Nano 11, 1 (2016).

32. Soleymani, L. et al. Hierarchical nanotextured microelectrodes overcome the molecular transport barrier to achieve rapid, direct bacterial detection. ACS Nano 5, 3360-3366 (2011).

33. Vasilyeva, E. et al. Direct genetic analysis of ten cancer cells: Tuning sensor structure and molecular probe design for efficient mrna capture. Angew. Chem. Int. Ed. 50, 4137-4141 (2011).

34. Ivanov, I. et al. Chip-based nanostructured sensors enable accurate identification and classification of circulating tumor cells in prostate cancer patient blood samples. Anal. Chem. 85, 398-403 (2013).

35. Lam, B. et al. Solution-based circuits enable rapid and multiplexed pathogen detection. Nature Communications 4 (2013).

36. Maier, S. Plasmonics: Fundamentals and Applications (Springer, 2007). URL http://www. springer. com/west/home/librarians/springer+news?SGWID=4-40157-22-173660338-0.

37. Leonhardt, U. Optical conformal mapping. Science 312, 1777-1780 (2006).

38. Pendry, J., Aubry, A., Smith, D. \& Maier, S. Transformation optics and subwavelength control of light. Science 337, 549-552 (2012). 
39. Giugni, A. et al. Hot-electron nanoscopy using adiabatic compression of surface plasmons. Nature Nanotechnology 8, 845-852 (2013).

40. Ip, A. H. et al. Hybrid passivated colloidal quantum dot solids. Nat Nano 7, 577-582 (2012). 